\title{
Ok, boomer. uno scontro generazionale combattuto (anche) a colpi di meme
}

\author{
Sara Giovine
}

PUBBLICATO: 25 GENNAIO 2021

\begin{abstract}
A nche a chi abbia scarsa dimestichezza con i social network sarà probabilmente capitato di imbattersi nella parola boomer (o peggio, di essere cosi interpellato/-a da una persona più giovane): si tratta infatti di una forma che in tempi recenti si è diffusa, anche in italiano, nell'uso dei giovani e giovanissimi per indicare in modo ironico, e spesso spregiativo, una persona più anziana che riveli atteggiamenti e modi di pensare da questi ritenuti ormai superati (specialmente dal punto di vista politico, sociale e tecnologico).
\end{abstract}

Nel suo significato proprio e originario, il termine boomer indica però più specificatamente una persona nata negli anni del cosiddetto "baby boom", l'esplosione demografica che ha interessato la maggior parte dei paesi occidentali al termine del secondo conflitto mondiale: la forma rappresenta infatti la riduzione del termine inglese baby boomer (derivato del composto baby boom, letteralmente 'esplosione (di nascite) di bambini' con l'aggiunta del suffisso -er proprio dei nomi d'agente), con il quale in diversi paesi del mondo sono appunto comunemente indicati i 'figli' di tale stagione di intenso sviluppo economico e forte incremento demografico, in particolare i nati tra il I946 e il I964.

Secondo la ricostruzione offerta dai principali strumenti lessicografici inglesi, tra cui l'Oxford English Dictionary, e da alcuni articoli dedicati all'argomento (per esempio questo), tale denominazione sarebbe stata coniata all'inizio degli anni Sessanta negli Stati Uniti, non a caso uno dei paesi maggiormente interessati dal fenomeno dell'incremento demografico del secondo dopoguerra: la prima attestazione di baby boomer risale in particolare al gennaio del I963, quando compare nel titolo di un articolo pubblicato sul quotidiano statunitense "Daily Press" (Leslie J. Nason, Baby Boomers, Grown Up, Storm Ivy-Covered Walls, "Daily Press", 28/I/I963, p. II), che approfondisce la questione del vertiginoso incremento delle iscrizioni nelle università americane come conseguenza del raggiungimento della maggiore età da parte dei molti giovani nati nell'immediato dopoguerra. Nel corso del successivo decennio si assiste alla crescente diffusione nell'uso del termine, di cui si moltiplicano le occorrenze in periodici e quotidiani, dove viene impiegato come nome-etichetta della nuova generazione di giovani americani che proprio in quegli anni stanno raggiungendo la maturità: alla rapida affermazione della forma potrebbe aver contribuito anche il fatto che alla sua base vi è il composto baby boom, attestato nell'inglese americano nel significato di temporaneo e marcato incremento della natalità' già dalla fine dell'Ottocento (dal i 880 secondo l'OED) e dunque ormai da tempo consolidato nella lingua comune, ma che solo a partire dagli anni Cinquanta del Novecento comincia a essere impiegato con specifico riferimento allincremento demografico del secondo dopoguerra.

Il sostantivo baby boomer comincia presto a diffondersi come prestito integrale dall'inglese anche in molte lingue europee, soprattutto in articoli e pubblicazioni che descrivono la realtà statunitense: in italiano le prime occorrenze del termine (attestato anche nella variante minoritaria con grafia univerbata babyboomer e in quella analitica, con i due elementi componenti separati da un trattino, baby-boomer) risalgono alla metà degli anni Ottanta, in articoli pubblicati sui maggiori quotidiani nazionali che appunto approfondiscono le ultime novità della scena politica e socio-culturale 
americana. La natura di prestito del termine è spesso segnalata, oltre che dall'impiego delle virgolette, anche dalla marca -s del plurale e dalle iniziali maiuscole. Per esempio:

Del resto, è anche difficile prevedere fino a che punto Mondale potrà essere vulnerabile a causa del suo veteropopulismo tanto indigesto agli "Yuppies", "Yumpies", "Baby Boomers", o a causa del suo triste periodo vicepresidenziale con lo sfortunato presidente Carter (la negativa immagine della "Carter connection"). (Alberto Ronchey, Un boom per il presidente, "la Repubblica", 3/5/1984, p. 3)

I tempi dei jeans e dei blusotti, dei capelli lunghi unisex e dei pasti consumati in piedi appartengono a un passato senza ritorno per questi protagonisti della generazione chiamata da alcuni la "Generazione di Gary Hart" e da altri la "Generazione di me stesso". Fanno parte dei Baby Boomers nati nel boom di bambini che segui la fine della guerra, all'ingrosso tra il 1945 e il I964, ma soprattutto sono i giovani che hanno adesso tra i 25 e 35 anni, sono stati all'Università, hanno lavorato negli uffici e costituiscono una ventina di milioni di votanti, un boccone quindi goloso per i politicanti. (Fernanda Pivano, In America é l'ora degli Yuppies, "Corriere della Sera", 2/2/1985, p. 3)

Nella seconda metà degli anni Ottanta e per tutti i successivi anni Novanta la forma, pur vantando una discreta circolazione (63 occorrenze di "baby boomer" nell'archivio della "Repubblica" e i7 in quello del "Corriere della Sera" in tale intervallo temporale), continua a essere impiegata pressoché esclusivamente in riferimento alla realtà statunitense e di norma accompagnata dalla spiegazione del suo significato; è necessario attendere l'avvento del nuovo millennio per assistere a una maggiore affermazione della parola e a un suo impiego esteso nel significato più generico di persona nata tra il I946 e il I964, indipendentemente dal paese di provenienza (e dunque non più con esclusivo riferimento alla popolazione americana). Nel primo ventennio del Duemila se ne contano, in tale più ampio significato, ben 455 esempi nell'archivio della "Repubblica", i23 in quello del "Corriere della Sera" e 76 in quello della "Stampa" (i cui articoli in questo momento, a causa del temporaneo blocco dell'archivio storico, sono però consultabili solo a partire dal 2006), per esempio:

Attraverso una ricerca che muove dai primi anni Sessanta e approda ai primi anni Ottanta, un coetaneo dei ragazzi di Giordana, già militante di Lotta continua, oggi docente di Storia contemporanea all'Università di Teramo, scava nelle vicende dell'Italia contemporanea per spiegare una tragedia: la tragedia per cui una scheggia di italici baby boomer, distaccatasi dal corpo della loro generazione, volle seminare all'intorno morte e terrore. (Sergio Luzzatto, Il '68 e le grandi illusioni di un Paese mancato, "Corriere della Sera", 28/9/2003, p. 33)

Il problema è che gli analisti della banca d'affari americana sottolineano come la generazione dei babyboomers europei tenda oggi a vestirsi servendosi indifferentemente da grandi magazzini a sconto come dalle boutique delle grandi firme ma che la vera discriminante è il prezzo. (s.n., Lumh pronta al pieno di utili ma Fendi resta ancora in rosso, "la Repubblica", sez. Economia, 9/3/2005, p. 35)

Il cambiamento è infatti il tema sociale più pressante dello sviluppo dei nostri Paesi occidentali, e in questo passaggio, i baby boomer fanno la parte del tappo, dell'élite, della conservazione insomma. (Lucia Annunziata, Good bye Sessantotto, Stampa.it, sez. Opinioni, 31/12/2007)

$\mathrm{Nel} 2003$ la forma è inoltre scelta come titolo di un fortunato volume autobiografico, scritto a più mani da quattro autrici che ripercorrono le loro vicende personali sullo sfondo dei maggiori avvenimenti storici e delle trasformazioni culturali dell'Italia del secondo Novecento (Rosi Braidotti, Roberta Mazzanti, Serena Sapegno, Annamaria Tagliavini, Baby Boomers. Vite parallele dagli anni Cinquanta ai cinquant'anni, Firenze, Giunti). Nello stesso anno il sostantivo è registrato nel primo supplemento del GRADIT dedicato alle Nuove parole italiane dell'uso e nel repertorio di Neologismi quotidiani di Giovanni Adamo e Valeria Della Valle, a ulteriore conferma della crescente diffusione 
della parola, che negli anni successivi viene registrata anche da alcuni dizionari sincronici (tra cui il Garzanti e il Devoto-Oli). All'effettivo consolidamento della voce quale nome-etichetta di una generazione ha però contribuito soprattutto la sua adozione da parte di autorevoli istituti di ricerca, quali l'Istat in Italia e il Pew Research Center negli Stati Uniti, che in anni recenti hanno fissato gli estremi cronologici delle diverse generazioni e stabilito una denominazione convenzionale per ciascuna di esse: secondo gli studiosi di demografia, la generazione dei Baby Boomer, l'unica peraltro ad aver ricevuto una denominazione ufficiale da parte dell'United States Census Bureau (l'agenzia governativa statunitense che si occupa di censimento è quella delle persone nate tra il I946 e il I964; è preceduta dalla Generazione silenziosa (in inglese Silent Generation), in italiano nota anche come la Generazione della ricostruzione, in quanto costituita dai nati tra il I926 e il I945, che hanno permesso la ripresa del paese al termine della guerra; ed è seguita nell'ordine dalla Generazione X o Generazione di transizione (i nati tra il 1965 e il I980), dalla Generazione Y o dei Millennial (i nati all'incirca dal I98I al I995, su cui si veda la scheda di Maria Cristina Torchia), e infine dalla Generazione Z o Generazione delle reti, formata dai giovani nativi digitali nati a partire dal I996-I997.

In inglese (cosi come nelle altre lingue) l'uso della forma baby boomer, sia nella lingua corrente, sia in testi specialistici di sociologia e demografia, è stato ben presto affiancato da quello della sua variante ridotta boomer: la prima attestazione registrata dall'OED, rilevata in un articolo del quotidiano canadese "Toronto Star", è datata 1976 ("The young always go through a period of rejecting the parent generation's values and structures, and the boomers did it more completely than ever" [i giovani attraversano sempre un periodo di rifiuto dei valori e delle strutture della generazione dei genitori e i boomers l'hanno fatto in maniera più completa che mai], "Toronto Star", Canad. Suppl., 4/3, 24/I/1976), quindi successiva di circa un decennio alla prima occorrenza di baby boomer (I963). E tuttavia plausibile che le prime occorrenze della forma, nata appunto come riduzione informale di baby boomer (come segnalato dal Cambridge Dictionary, che la marca come variante "US informal"), siano in realtà di poco successive ai primi esempi d'uso del sostantivo nella sua forma 'estesa', e che l'abbreviazione sia quindi già in uso verso la metà degli anni Sessanta: a tale altezza cronologica il sostantivo boomer risulta infatti già impiegato in un testo italiano, in particolare in una poesia composta tra il 1964 e il I968 da Nanni Balestrini, uno dei maggiori esponenti della neoavanguardia, che nelle sue opere ricorre spesso alla tecnica del collage e a prestiti da altre lingue:

e lo sciopero de / i boomers la nuova situazione il / culto anglo-americano le conseguenze la pace security act [...]. (Nanni Balestrini, [sulle acque economiche], in Ma noi facciamone un'altra. Poesie 19641968, Milano, Feltrinelli, I968, p. 150).

In italiano, se si esclude l'occorrenza isolata rilevata nei versi di Balestrini, è però necessario attendere l'inizio degli anni Novanta per avere le prime sporadiche attestazioni di boomer, rilevate quasi esclusivamente in saggi specialistici di argomento sociologico o in articoli delle principali testate nazionali dedicati all'attualità politica e sociale degli Stati Uniti: diverse in particolare le occorrenze che fanno riferimento al neoeletto presidente americano, Bill Clinton, il primo ad appartenere alla generazione del cosiddetto baby boom, o quelle legate ai primi studi che teorizzano l'influsso dei valori e dello stile di vita della generazione di appartenenza sulle abitudini di consumo di una persona. Per esempio:

Sarebbe inutile cercare la carta d'identità dei giornalisti e dei capiredattore che hanno stilato la geremiade contro i giovani, sono tutti boomer, marchiati dal narcisismo di chi ritiene di avere il monopolio dell'impegno e dell'altruismo. (Barbara Stefanelli, Clinton attento, c'è la «generazione X», "Corriere della Sera", sez. Esteri, 5/I/1993, p. 7). 
I Boomers dominano tuttora la scena. Sono quelli che hanno dato vita sia agli hippies sia agli yuppies. Quelli che hanno rotto gli schemi con sesso, droga e rock 'n' roll. Il loro principio di vita è stato ed è: fare esperienze. (Serena Zoli, L'età è l'anima del mercato, "Corriere della Sera", 29/9/1998, p. 35).

Gli ultimi boomers (nati nel 1964) raggiungono i zo anni nel I994: ma come può - dice Ritchie (1995) - un boomer essere così giovane da non potersi ricordare del Vietnam, né dei days of rage al tempo del liceo? (Giuseppe A. Micheli, Effetto generazione: cinquant'anni di trasformazioni demografiche in Italia dal dopoguerra a oggi, Roma, Carocci, 1999, p. 86).

Solo a partire dai successivi anni Duemila, la forma comincia a godere di una circolazione relativamente più ampia, con una parabola di diffusione che quindi grosso modo coincide con quella della variante 'estesa' baby boomer, alla quale viene nella maggior parte dei casi affiancata, probabilmente per evitare più ripetizioni dello stesso termine allinterno del medesimo testo: una ricerca negli archivi dei maggiori quotidiani nazionali (condotta il 28/I2/2020) restituisce circa I $_{5}$ occorrenze di boomer e ig del plurale boomers negli articoli della "Repubblica" ( 2 del 2005 e tutti gli altri successivi al 20I0), I esempi di boomer e iz di boomers nella "Stampa" (tutti posteriori al 20I0), II di boomer e 5 di boomers nel "Corriere" (di cui 4 degli anni Novanta, uno del 20o6, uno del 2009 e tutti gli altri successivi al 20I4). Per esempio:

I primi boomers avevano un anno quando apparvero i primi programmi in tv per bambini, ne avevano I7 quando Kennedy fu assassinato, 23 quando si radunarono a Woodstock, 36 all'inizio del rialzo della Borsa degli anni '8o. (Jerry Adler, I baby boomers, giovani a sessant'anni, "la Repubblica", sez. Politica estera, $7 /$ II $/ 2005$, p. I7)

Il passaggio del testimone dai "boomers" ai "millennials", però, è significativo e anche controverso. I nati fra il 1946 e il 1964, infatti, si vantano di aver cambiato la società, inventando l'impegno politico. I "millennials", invece, vengono descritti spesso come freddi, distaccati, egoisti, immersi nella realtà digitale e disinteressati alla società che li circonda. (Paolo Mastrolilli, Addio baby boomers, il mondo é dei millennials, Stampa.it, sez. Cultura, 21/r/2015)

Lo ha appena fatto la multinazionale americana Kelly Services con il "Global workforce index", che ha intervistato I64 mila lavoratori di 68 paesi. In particolare gli over 53 (baby boomer) e gli under 35 (millennial), tra i quali prosperano i pregiudizi. Il $56 \%$ dei boomer italiani è convinto che i millennial tendano a "pretendere profitti e riconoscimenti immediati" e il $34 \%$ che non abbiano alcuna volontà di "dedicare il tempo necessario al lavoro duro o a portare a termine i loro compiti". (Enzo Riboni, Generazioni in conflitto in tre casi su quattro, "Corriere della Sera", sez. Economia, 27/6/2017, p. 33)

Nonostante il discreto incremento delle occorrenze registrate nei giornali, soprattutto nel secondo decennio dei Duemila, l'uso della forma resta tuttavia circoscritto a testi specialistici o ad articoli che mettano a confronto gusti e abitudini di tale generazione con quelle dei più giovani; e almeno fino al 20I8-2019 il termine continua a essere accompagnato dalla spiegazione del suo significato o evidenziato tra virgolette, a segnalarne la natura di prestito, non ancora del tutto acclimatato nella lingua corrente. Le cose cominciano a cambiare a partire dal 2019, quando boomer inizia a diffondersi nell'uso dei più giovani, soprattutto in rete e nei social media, per indicare in senso ironico e spesso dispregiativo non solo gli appartenenti alla generazione del baby boom, ma piu in generale qualsiasi persona più anziana che dimostri atteggiamenti e modi di pensare ormai superati, per esempio opponendo resistenza al progresso tecnologico e sociale, negando l'esistenza del cambiamento climatico o sminuendo l'importanza degli attuali dibattiti sull'identità (etnica, di genere o legata allorientamento sessuale). La nuova accezione della parola, sviluppatasi per estensione a partire dal suo significato originario, viene efficacemente illustrata, tra gli altri, dalla scrittrice e giornalista italiana Giulia Blasi in un volume del 2020 che si rivolge alla generazione dei più giovani (la cosiddetta 
Generazione Z), approfondendo le questioni del femminismo, dell'uso della rete e del conflitto generazionale:

Il significato che diamo a "boomer" nel linguaggio dei social non è legato all'età. Boomer è uno stato dell'anima. Boomer non è chi ha compiuto un tot di anni, ma chi non sa mettere a sistema tutti $\mathrm{i}$ chilometri che ha percorso. [...] Boomer è essere sospettosi verso ogni novità, rigidi con gli altri, inconsapevoli del proprio privilegio e infastiditi da chiunque lo metta in discussione. Boomer è chi non accetta che le persone possano avere identità e orientamenti diversi da uomo-donna, etero-gay, e che possano rivendicarne il riconoscimento alla luce del sole. Boomer è chi all'alba del 2020 ancora tratta il femminismo come un attacco agli uomini e rifiuta di riconoscerne l'importanza come filosofia di liberazione. (Giulia Blasi, Rivoluzione Z. Diventare adulti migliori con il femminismo, Milano, Rizzoli, 2020).

La fortuna della parola nella sua più recente accezione è decretata soprattutto dalla diffusione virale, in diversi paesi del mondo, di diversi meme, ossia di immagini di norma associate a una frasetormentone (su cui si veda la scheda di Vera Gheno), che in questo caso contiene al suo interno la nostra voce e che a partire dal 2019 ha goduto di una popolarità crescente in rete e su social network come Twitter e TikTok: si tratta di "Ok boomer", un'espressione usata soprattutto da giovani e adolescenti della Generazione $\mathrm{Z}$ come risposta sarcastica alle critiche e alle osservazioni paternalistiche delle persone più anziane, nella maggior parte dei casi appunto della generazione dei cinquanta-sessanta-settantenni. Secondo la ricostruzione proposta dal portale "Know your meme" (un database che raccoglie piu di 4.000 meme, ripercorrendone origine e storia) e da un articolo del "New York Times" dell'ottobre del 2019 (Taylor Lorenz, 'OK Boomer' Marks the End of Friendly Generational Relations, "The New York Times", 29/Io/2019), l'espressione, pur precedentemente attestata, avrebbe cominciato a essere impiegata negli Stati Uniti nel corso del 20I8, per rispondere ai post pubblicati su Twitter da politici e altre personalità critiche verso le generazioni più giovani, e sarebbe poi divenuta sempre più popolare nel corso dell'anno successivo, soprattutto in seguito alla diffusione, nell'ottobre del 20r9, di un video di TikTok che affianca le accuse rivolte da un uomo non identificato ai millennial e ai giovani della Generazione Z, ritenuti idealisti e immaturi, e la reazione a tali accuse di un adolescente, che si limita a sollevare un quaderno con la scritta "ok boomer". Nelle settimane successive la frase viene quindi rilanciata a più riprese sui social network e dagli Stati Uniti il suo uso comincia a estendersi anche in altri paesi del mondo; inoltre la pubblicazione del già citato articolo di Taylor Lorenz, che chiarisce l'origine e il significato del meme, su un quotidiano di prestigio internazionale come il "New York Times", fa sì che l'espressione divenga argomento di discussione anche al di fuori del ristretto ambito d'uso dei giovani e della rete. La notorietà mediatica e internazionale del meme viene poi ulteriormente accresciuta in seguito all'intervento parlamentare del 4 novembre 2019 di una giovane deputata neozelandese, Chlöe Swarbrick, che ricorre all'espressione per zittire un collega più anziano che aveva cercato di interromperla: la notizia della vicenda viene riportata da diversi telegiornali e quotidiani internazionali e nei giorni successivi si moltiplicano, anche in italiano, gli articoli che spiegano il significato di "ok boomer", che alla fine dell'anno è tra le dieci parole ed espressioni più rappresentative del 2019 segnalate da Lucia Corbolante nel suo blog "Terminologia etc.". Per esempio:

Oggi "Ok, Boomer" è diventata una sorta di risposta-reazione alle paternali antipatiche e distanti che i membri della "Generazione Z" (cioè nati tra la fine degli anni 'go e la fine dei 'oo) devono sorbirsi dai "Baby boomer" (cioè nati tra la metà degli anni '40 e '60). È un'espressione ma soprattutto un meme nato su TikTok e su Twitter, finito sul New York Times e che ormai è ovunque. Praticamente una guerra tra nonni e nipoti, come se all'ennesima borbottata sui soldi di Zio Paparone, Qui Quo e Qua rispondessero insieme: "Ok, Boomer!". (Valerio Coletta, Cosa vuol dire "Ok, Boomer", la frase che state sentendo ovunque, Esquire.com, 8/II/2019) 
"Boomer" diventa un'offesa nel momento in cui in una sede istituzionale, come un parlamento, una 25enne apostrofa un collega più anziano con "ok, boomer", zittendolo e dandogli, di fatto, del vecchio rimbambito, perché il significato, il sottotesto, è questo. "Ok, dai, lasciami finire, vecchio babbione" (e scusate il termine babbione, che fa molto boomer ma evita sinonimi più coloriti...). (Alberto Scotti, Se qualcuno ti dice "ok boomer" devi sapere che... (da dove arriva questa espressione virale e cosa significa), ilfattoquotidiano.it, sez. Attualità, I7/II/2019)

Anche in italiano l'espressione gode quindi di una discreta circolazione, soprattutto in rete e nei principali mezzi di comunicazione (5I.400 occorrenze della stringa di ricerca "ok boomer" nelle pagine italiane di Google) e ciò concorre, di riflesso, a una maggiore diffusione anche del sostantivo boomer (divenuto quasi una parola-simbolo dello scontro generazionale), che nel 2020 è registrato dal portale Treccani nella sezione "Neologismi 2020" insieme alla variante estesa baby boomer. Il nuovo significato della forma tuttavia non è ancora accolto da alcun dizionario sincronico: solo il tempo potrà dirci se si tratta di una 'moda' linguistica temporanea, legata all'attuale diffusione dell'espressione (e all'attuale scontro generazionale), destinata quindi a esaurirsi nei prossimi anni, o se l'uso di boomer arriverà a consolidarsi anche nella lingua corrente, divenendo a tutti gli effetti parte del lessico italiano.

Per quanto riguarda infine la grafia del termine, che in italiano oscilla tra la variante con l'iniziale minuscola e quella con la maiuscola, va detto che analogamente a quanto osservato per il sostantivo millennial, trattandosi non di un nome proprio, ma di un nome comune, che identifica una classe di individui e non un singolo, boomer non richiede, secondo l'attuale norma ortografica, l'uso della maiuscola. Quanto alla corretta declinazione al plurale del termine, che oscilla tra la forma invariabile ("i boomer") e quella flessa secondo le regole morfologiche dell'inglese, che prevedono l'aggiunta della desinenza -s ("i boomers"), è forse preferibile optare per il plurale invariabile, che è del resto anche quello attualmente prevalente nell'uso ( 5.800 risultati della stringa di ricerca "i boomer" e 9.200 di "dei boomer" in Google Italia, contro i ro.50o di "i boomers" e i 5.790 di "dei boomers"), oltre a essere in linea con il trattamento di norma riservato alle parole straniere da tempo attestate nella nostra lingua.

\section{Cita come:}

Sara Giovine, Ok, boomer: uno scontro generazionale combattuto (anche) a colpi di meme, "Italiano digitale", 2021, XVI, 2021/1 (gennaio-marzo)

DOI: 10.35948/2532-9006/2021.5463

Copyright 2021 Accademia della Crusca

Pubblicato con licenza creative commons CC BY-NC-ND 